Analytical Methods

\title{
Authentication of a Turkish traditional aniseed flavoured distilled spirit, raki
}

\author{
Dila Yucesoy, Banu Ozen* \\ Izmir Institute of Technology, Department of Food Engineering, Urla, Izmir, Turkey
}

\section{A R T I C L E I N F O}

\section{Article history:}

Received 10 June 2011

Received in revised form 19 April 2012

Accepted 4 April 2013

Available online 20 April 2013

\section{Keywords:}

Raki

Aniseed spirit

Adulteration

Chemometrics

Mid-infrared spectroscopy

\begin{abstract}
A B S T R A C T
Consumption of traditional aniseed alcoholic beverage, raki, adulterated with methanol results in deaths, therefore, its detection is an important issue. In this study, mid-infrared spectra of pure and methanol adulterated $(0.5-10 \%(\mathrm{vol} / \mathrm{vol}))$ raki samples were collected with an attenuated total reflectance attachment of a Fourier-transform infrared spectrometer. Principal component analysis was used to discriminate pure and adulterated raki samples, then, a partial least square model was constructed to determine the adulterant methanol content in raki using mid-IR spectral data. A minimum threshold level of $0.5 \%$ methanol in raki samples was successfully detected. A good prediction model for determination of methanol adulteration ratio in raki samples was also constructed $\left(R^{2}=0.98\right.$ and $\left.R P D=8.35\right)$.
\end{abstract}

(C) 2013 Elsevier Ltd. All rights reserved.

\section{Introduction}

Aniseed flavoured distilled spirits are produced in different Mediterranean countries and are known with various names such as anis, arak, pastis, ouzo, sambuco and zivania (Anli \& Bayram, 2010). Raki, a traditional Turkish alcoholic beverage, also belongs to this group. It is the second most produced alcoholic beverage in Turkey $(20,083 \mathrm{~L}$ absolute alcohol in 2009 (TAPDK, 2010)) and is described in Turkish Food Codex (2005) as a spirit that must be produced in Turkey by double distillation of suma or suma mixed with agricultural-based ethanol and flavoured with aniseed (Pimpinella anisum). Suma is produced mainly from raisins, molasses and/or grape must and is a distillate with a maximum $94.5 \%$ ethanol content. Suma can be also mixed with ethanol from an agricultural origin and this final mixture must contain at least $65 \%$ suma. Suma or suma-ethanol mixture is distilled in copper alembics with a maximum capacity of $5000 \mathrm{~L}$ and three groups of distillates, head, medium and tail, are separated (Anli \& Bayram, 2010). Medium distillate is used for raki manufacture and diluted to $45 \%$ alcohol. After the addition of sugar product is stored for at least one month before filling.

Alcoholic beverages could be mixed with ethanol, methanol, water and aromatic compounds to gain illegal profit (Pontes et al., 2006). Consumption of the excess amounts of some of these adulterants could cause severe health problems. Severe metabolic disturbances, blindness, permanent neurologic dysfunction and death are the results of methanol ingestion. Although methanol it-

\footnotetext{
* Corresponding author. Tel.: +90 232750 6319; fax: +90 2327506196 .

E-mail address: banuozen@iyte.edu.tr (B. Ozen).
}

self is harmless, its metabolites are toxic and its consumption causes methanol poisoning in both humans and animals. Methanol spreads rapidly into the body via all routes. After dispersion in body compartments, methanol is firstly digested into formaldehyde by alcohol dehydrogenase enzyme. Formaldehyde is thirtythree times more toxic than methanol, and it is speedily converted into formic acid by aldehyde dehydrogenase enzyme. Finally, formic acid is metabolised into carbon dioxide then water is moved away from body (Kapur, Vandenbroucke, Adamchik, Lehotay, \& Carlen, 2007). Lethal dose of methanol in humans is related to age, sex, metabolic rate and methanol blood level. It was estimated that consumption of $50-75 \mathrm{~g}$ of methanol causes deaths due to methanol intoxication; however, there are also reports showing that toxication level could be as low as $11.5 \mathrm{~g}$ (Di Maio \& Di Maio, 2001; Fidan, Denli, \& Anli, 1996). Many death cases related to methanol intoxication due to consumption of fake raki have been reported in Turkey. According to literature, the total number of deaths due to methanol poisoning which was arisen from consumption of adulterated raki, has been more than 300 from 1990 to 2011 (Eke, Buyuk, Dinc, \& Citici, 2007; Gulmen, Meral, Hilal, Akcan, \& Cekin, 2006; Yaycı \& Inanici, 2005). Generally, chromatographic methods are applied for the detection of adulteration of alcoholic beverages (Savchuk et al., 2001; Yilmaztekin \& Cabaroglu, 2011). However, there has been an increasing interest towards the development of rapid techniques such as the use of different spectroscopic methods. Front face fluorescence spectroscopy was used in differentiation of brandies and wine distillates (Sadecka, Tothova, \& Majek, 2009). Zivania, a traditional Cypriot aniseed flavoured distilled beverage, was characterised by NMR spectroscopy and the data was used satisfactorily to differentiate zivania from 
other similar beverages (Petrakis et al., 2005). Distilled beverages, whiskey, brandy, rum and vodka were classified successfully using near infrared spectroscopy and verification of the mixing of these beverages with $5-10 \%$ of ethanol, methanol and water was also achieved with the same technique in combination with chemometric analysis (Pontes et al., 2006).

Mid-infrared (mid-IR) spectroscopy in combination with chemometric techniques has become a very effective tool in detecting the adulteration of various food products such as honey, different types of oils, coffee and fruit juices (Briandet, Kemsley, \& Wilson, 1996; Gurdeniz \& Ozen, 2009; Kelly \& Downey, 2005; Sivakesa \& Irudayaraj, 2001). There are also examples of the application of mid-IR spectroscopy in literature for authentication of wines from different origins as well as detection of its mixing with adulterants such as glycerol (Dixit, Tewari, Cho, \& Irudayaraj, 2005; Tarantilis, Troianou, Pappas, Kotseridis, \& Polissiou, 2008).

This study aims at detecting and quantifying the adulteration of raki with methanol with mid-IR spectroscopy in combination with chemometric techniques.

\section{Materials and methods}

\subsection{Samples}

Raki samples were obtained from local markets and cover most of the brand names and their sub-brands produced in Turkey. More than one sample from some brands was also included into the sample set. Two ouzos, aniseed drink from Greece, were also tested as samples. Totally, 34 samples were analysed. Samples, their raw materials and process types according to descriptions on the labels and information obtained from producers are listed in Table 1 . The raki samples were stored in their original bottles at $4{ }^{\circ} \mathrm{C}$. The lids of the bottles were covered further with parafilm in order to avoid alcohol volatilisation.

Six representative raki samples $(1,10,15,16,17,24)$ were selected and they were adulterated with $0.5-10 \%(\mathrm{v} / \mathrm{v})$ methanol (99.9\%, Sigma). Spectra of all pure and adulterated samples were obtained with a Fourier-transform infrared (FT-IR) spectrometer.

Table 1

List of raki samples.

\begin{tabular}{|c|c|c|c|}
\hline Sample number & Production process & Grape type & Alcohol content (\%) \\
\hline 1 & Distillated twice & Fresh-dried & 45 \\
\hline 2 & Distillated twice & Fresh-dried & 45 \\
\hline 3 & Distillated twice & Dried & 40 \\
\hline 4 & Distillated twice & Fresh & 45 \\
\hline 5 & Distillated twice & Dried & 45 \\
\hline 6 & Distillated twice & Dried & 43 \\
\hline 7 & Distillated twice & Dried & 45 \\
\hline 8 & Distillated twice & Fresh-dried & 45 \\
\hline 9 & Distillated twice & Dried & \\
\hline 10 & Distillated twice & Dried & 45 \\
\hline 11 & Distillated twice & Dried & 45 \\
\hline 12 & Distillated twice & Dried & 45 \\
\hline 13 & Distillated twice & Fresh-dried & 43 \\
\hline 14 & Distillated twice & Fresh & 50 \\
\hline 15 & Distillated thrice & Dried & 47 \\
\hline 16 & Distillated twice & Dried & 45 \\
\hline 17 & Distillated twice & Fresh & 47 \\
\hline 18 & Distillated twice & Dried & 45 \\
\hline 19 & Distillated twice & Dried & \\
\hline 20 & Distillated thrice & Dried & 45 \\
\hline 21 & Distillated thrice & Fresh & 45 \\
\hline 22 & Distillated twice & Dried & 43 \\
\hline 23 & Distillated twice & Dried & 43 \\
\hline 24 & Distillated twice & Dried & 45 \\
\hline 25 & Distillated twice & Dried & 45 \\
\hline 26 & Distillated twice & Dried & 45 \\
\hline 27 & Traditional ouzo & & 38 \\
\hline 28 & Traditional ouzo & & 38 \\
\hline
\end{tabular}

\subsection{Mid-IR analysis}

All infrared spectra $\left(4000-650 \mathrm{~cm}^{-1}\right)$ were obtained using a Perkin Elmer Spectrum 100 FT-IR spectrometer (Perkin Elmer Inc., Wellesley, MA) with a deuterated tri-glycine sulphate (DTGS) detector. Samples were placed on a horizontal attenuated total reflectance (HATR) sampling accessory with a ZnSe crystal (45 deg. Trough Plate) and covered with a lid to prevent evaporation of alcohol. The number of scans for each spectrum was 64 . The resolution of the instrument was set to $4 \mathrm{~cm}^{-1}$. ZnSe crystal was cleaned with ethanol and water in between sample runs. At least three measurements were collected for each sample.

\subsection{Multivariate analysis}

Spectral data obtained with an FT-IR spectrometer were analysed by using multivariate statistical techniques with SIMCA software (SIMCA P-10.5 Umetrics Inc. Sweden). Whole spectrum (4000-650 $\left.\mathrm{cm}^{-1}\right)$ and also three useful spectral intervals selected to avoid interferences and noises were used in the data analysis. These selected spectral regions were 1565-965, 1900-1700 and $3040-2800 \mathrm{~cm}^{-1}$ (Lachenmeir, 2007; Versari, Parpinello, Scazzino \& Rio, 2010).

\subsubsection{Pre-treatment of data}

Spectral data set was transformed into suitable form by preprocessing techniques of scaling and centering. Raki samples constitute the columns and the variables (spectral data) are located in the rows. Pre-processing can be improved by using mathematical procedures including signal corrections, some various derivative forms and using some filtering techniques such as wavelet compression of spectra (WCS), wavelet in combination with orthogonal signal correction (WOSC) and orthogonal signal correction in combination with wavelet (OSCW) (Casela, Sinelli, Oliveri, Valentina Di Egidio, \& Silvia Lanteri, 2010). For obtaining better classification with principal component analysis (PCA), wavelet compression of spectra (WCS) was preferred as spectral filtering technique in this study. Daubechies-10 function wavelet was chosen and confidence interval was set to 99.95\%. De-noise and sophisticated signals are compressed with WCS technique which depends on retaining the significant coefficients from the representation of the data in the new ordinate system (Eriksson, Johanson, \& Wold, 2001). For partial least square (PLS) analysis, OSCW technique was applied.

\subsubsection{Classification}

Spectral data of pure and methanol adulterated raki samples were analysed by PCA for classification. The data matrix consists of observations represented by raki samples and variables represented by FT-IR spectral data. The classification method is used to determine the group of raki samples based on the similarities among members of the same data class (Pontes et al., 2006). Assignment of the sufficient number of principal components (PCs) depends on the goodness of fit, which is represented by the parameter $R^{2}$, and predictive ability of the model (Eriksson, Johanson, \& Wold, 2001).

Score and Coomans' plots were constructed as part of classification analysis. When a grouping was observed in score plot then Coomans' plot was also used. The Coomans' plot compares the distance to the model results against the distance from the model centre for unknown samples of selected models (Esbensen, Guyot, Westad \& Houmoller, 2002). Coomans' plot comprises of four regions, two regions specify pure and adulterated classes defined by the models, common region indicates overlap of the two classes, and the other region indicates the observations far from two classes. 


\subsubsection{Quantification}

PLS was employed to determine adulteration rate of methanol adulterated raki using both whole and selected spectral ranges. PLS analysis was performed to establish a regression model for prediction of adulterant concentration (variable matrix Y) using FT-IR spectra (variable matrix X).

Obtained data set was randomly separated into two groups as calibration (2/3 of samples) and validation ( $1 / 3$ of samples) sets. The predictive ability of the model was expressed by some parameters and visualised with prediction plots of constructed models. These parameters are root mean square error of calibration (RMSEC), root mean square error of prediction (RMSEP), relative error of prediction (REP), residual predictive deviation (RPD) and the regression correlation coefficient $\left(R^{2}\right)$ both for calibration and validation models. RMSEC and RMSEP values are used to evaluate the performance in the prediction process. RMSEC refers to the calibration uncertainty that can be expected for predictions. A good model would have small value of RMSEC and RMSEP. Assessing the accuracy of the model can be also expressed using RPD value. Generally evaluating all these parameters gives an idea about the predictive efficiency of the model (Esbensen et al., 2002; Tamaki \& Mazza, 2011). RMSEC, RMSEP and RPD are defined with following equations:

RMSEC $=\sqrt{\frac{\sum_{i=1}^{n}\left(\hat{y}_{i}-y_{i}\right)^{2}}{n-2}}$

RMSEP $=\sqrt{\frac{\sum_{i=1}^{n}\left(\hat{y}_{i}-y_{i}\right)^{2}}{n-1}}$

$\mathrm{RPD}=\frac{\mathrm{SD}}{\mathrm{RMSEP}}$

$\mathrm{SD}=\sqrt{\frac{\sum_{i=1}^{n}\left(y_{i}-\bar{y}\right)^{2}}{n-1}}$

where $n$ is the number of samples used in each set; SD is standard deviation in each set, $y_{i}$ is the actual value for $i$-th sample, $\hat{y}_{i}$ is the predicted value determined by FT-IR for the same sample and $\bar{y}$ is the mean of each set. Criteria for $R^{2}$, RPD and slope used to evaluate the performance of predictive models are listed by Tamaki and Mazza (2011).

\section{Results and discussions}

Fig. 1 shows the typical spectra of the raki samples obtained in this study. All pure samples have similar spectra; however, there are changes in the intensity of some peaks depending on the sample. These differences are mainly for peaks at 3300, 2975-2900, 1640 and $1450-1250 \mathrm{~cm}^{-1}$. FT-IR spectra show absorption bands at different frequencies and these bands are attributed to the various functional groups. The two distinct absorption bands around $3600-3200$ and $1700-1565 \mathrm{~cm}^{-1}$ are associated with $\mathrm{O}-\mathrm{H}$ stretching vibrations. The peak at $3040-2800 \mathrm{~cm}^{-1}$ is due to the stretching vibration of $\mathrm{C}-\mathrm{H}$ bond. The peaks between 1565 and $965 \mathrm{~cm}^{-1}$ are in the fingerprint area where many different IR bands, including those corresponding to the vibrations of the $\mathrm{C}$ $\mathrm{O}, \mathrm{C}-\mathrm{C}, \mathrm{C}-\mathrm{OH}, \mathrm{C}-\mathrm{H}$, and $\mathrm{C} \equiv \mathrm{N}$ bonds occur. Bands at 2960$2850 \mathrm{~cm}^{-1}$ (C-H stretch) and 1083-1045 (C-O stretch) $\mathrm{cm}^{-1}$ are attributed to ethanol while various groups belonging to organic acids, carbohydrates and phenols provide peaks at fingerprint region of $1500 \mathrm{~cm}^{-1}$ and below. Specifically, it was reported that organic acids have peaks at $1150-1060 \mathrm{~cm}^{-1}$ (C-O stretch) and $1420-1320 \mathrm{~cm}^{-1}$ (O-H bend) and peaks around $1100-900 \mathrm{~cm}^{-1}$ belong to residual carbohydrates (Polshin et al., 2011). The region from 4000 to $3600 \mathrm{~cm}^{-1}$ and the spectral range from 965 to $650 \mathrm{~cm}^{-1}$ contain very little useful information (Fernandez \& Agosin, 2007; Lachenmeier, 2007). Therefore, in addition to whole spectral range, a selected range (1565-965, 1900-1700 and 3040$2800 \mathrm{~cm}^{-1}$ ) was also used in statistical analysis to reduce noises by eliminating areas with no peak and excluding $-\mathrm{OH}$ peaks which were very dominant.

The first aim of this study is discrimination of methanol adulterated and pure raki using PCA. Adulteration range that was used in this study was $0.5-10 \%(\mathrm{vol} / \mathrm{vol})$. Raki could be produced from fresh, dried or fresh and dried grapes. Either suma alone or suma and ethanol from an agricultural source could be used in distillation. Also, distillation could be applied twice or three times during production. Six raki samples which are representative of different raw materials and processing techniques were adulterated so that it would be possible to take into account of the differences between raki samples. IR spectra of methanol adulterated and pure

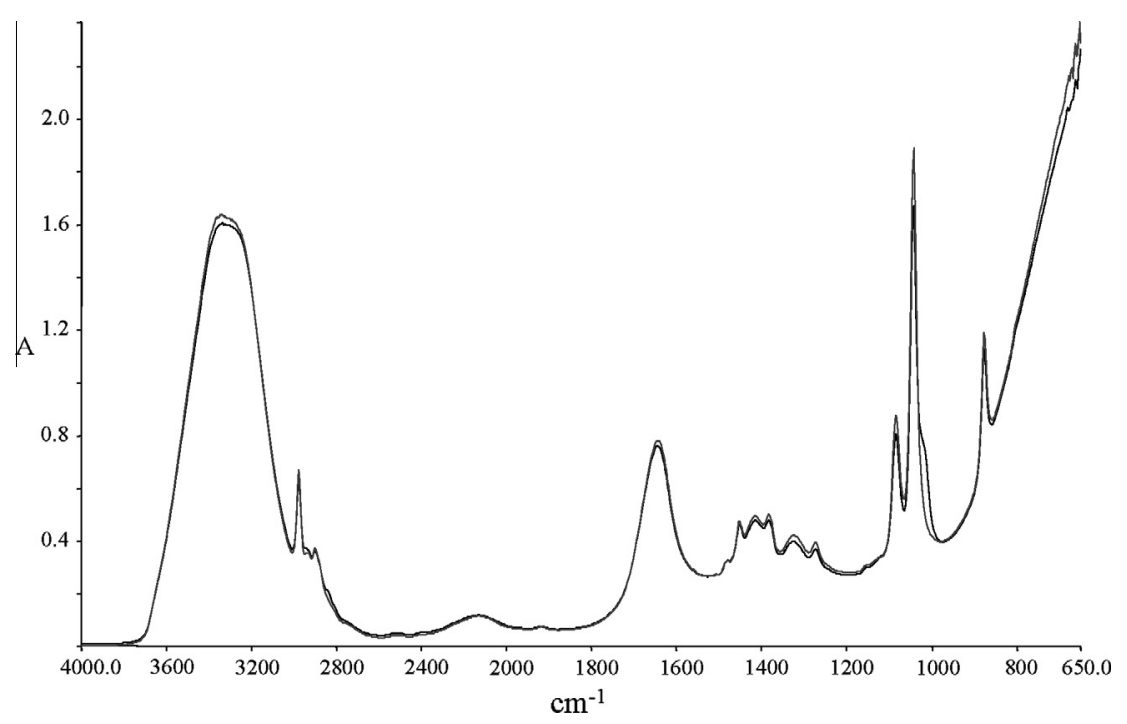

Fig. 1. Typical raki (gray line) and methanol adulterated (black line) mid-infrared spectra of raki. 
raki are provided in Fig. 1. As can be seen from spectra, distinctness arising from methanol content could be determined by comparing several regions. The first big difference corresponds to region around 3500 to $3200 \mathrm{~cm}^{-1}$ that can be attributed to $\mathrm{O}-\mathrm{H}$ stretching. Another difference in region $2900-2800 \mathrm{~cm}^{-1}$ arises from $\mathrm{C}-\mathrm{H}$ stretching and its source could be methyl groups. The other distinction between pure and adultered raki is in the fingerprint area (between 1565 and $965 \mathrm{~cm}^{-1}$ ). The differences between pure and adulterated sample spectra could be attributed to the absorbance bands specifically to methanol (spectrum not shown).

Both the whole and selected ranges of spectra were used for classification. WCS filtering was also applied to both cases. According to score plots (not shown), similar results were obtained using both the whole spectra and the selected range. Adulterated raki samples generally located on the left side of score plot while pure ones at the right side. There are some adulterated samples which are located in pure samples. When selected range of spectra was the data set 8 PCs were used to construct the model and 2 PCs explained $91.10 \%$ of the variance.

Since a separation was observed between pure and adulterated samples further analysis of data was performed with SIMCA class models and Cooman's plot is constructed. Cooman's plot for the classification of pure and adulterated raki samples shows good separation of adulterated raki with the ratio of $0.5 \%$ to $10 \%$ from pure samples (Fig. 2). By using whole spectral range (4000$650 \mathrm{~cm}^{-1}$ ), 12 PCs and 16 PCs were used to construct class models. Pure raki samples are located in the upper side of the plot and separated from adulterated raki samples and there were no samples in the common region. In addition, samples also show grouping between each other according to their adulteration ratio. Detection of adulteration in alcoholic beverages was investigated in other studies. In the study of Pontes et al. (2006); 69 samples of alcoholic beverages (whiskey, brandy, rum and vodka) were investigated to detect adulteration with water, ethanol or methanol by using near-infrared spectroscopy and chemometric methods. Successful results were also obtained for the verification of adulteration as a result of this study.

FT-IR in association with multivariate chemometric techniques was also employed to quantify the amount of methanol in adulterated raki samples. Results indicated that PLS model based on IR data was proven suitable as a practical analytical method for predicting adulterant content in raki samples in the volume fraction ranged from $0.5 \%$ to $10 \%$. Fig. 3 shows observed vs. predicted values of methanol concentration in raki for calibration and validation sets. RPD, slope and $R^{2}$ values were calculated for calibration and prediction sets to test the accuracy of the model. The accurate and similar prediction results were obtained by PLS using both whole and selected spectral ranges; for whole spectral range, $R_{\text {cal }} 2=0.9838, \quad R_{\mathrm{val}} 2=0.9914, \quad$ RMSEC $=0.44, \quad \operatorname{RMSEP}=0.35$, $\mathrm{RPD}=8.35$ and slope $=0.9$ values were obtained and for selected range $R_{\text {cal }} 2=0.9865, R_{\mathrm{val}} 2=0.9874$, RMSEC $=0.40$, RMSEP $=0.38$, $\mathrm{RPD}=8.29$, slope $=0.99$ values were calculated. According to criteria for RPD and slope values given by Tamaki and Mazza (2011), $R^{2}$ values greater than 0.9 and RPD values greater than 3 provide excellent predictions and slope between 0.9 and 1.1 means a very reliable model. Therefore, PLS regression of FT-IR spectral data provides excellent prediction and a very reliable model for the determination of methanol adulteration ratio in raki samples. Besides, small RMSEC and RMSEP values and small differences between these values indicated that the created models provide satisfactory prediction.

Mid-IR spectroscopy in combination with chemometrics techniques is successful in detection and quantification of methanol adulteration of a Turkish aniseed alcoholic beverage, raki and could be used as a rapid analysis tool especially for control laboratories of regulatory agencies. Lower detection limit of adulteration with this

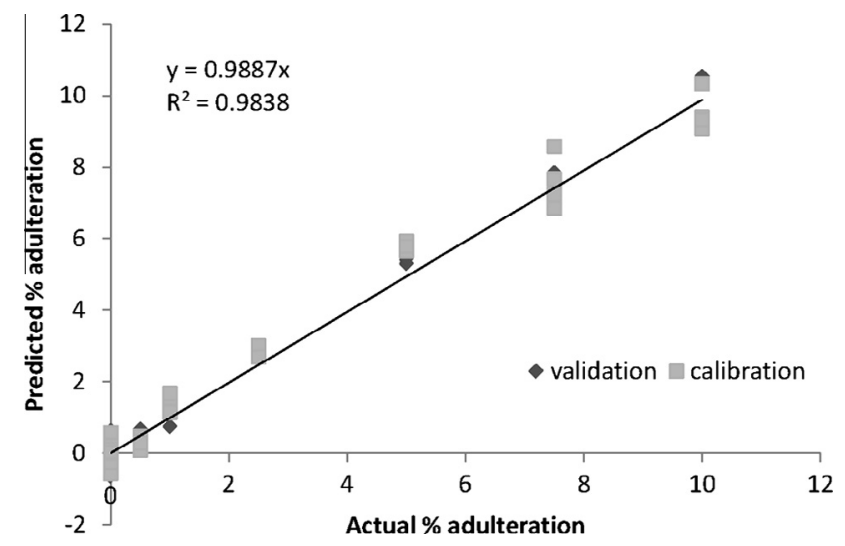

Fig. 3. OSCW PLS regression of actual vs. predicted adulteration of calibration and validation sets (whole spectral range: $4000-650 \mathrm{~cm}^{-1}$ ).

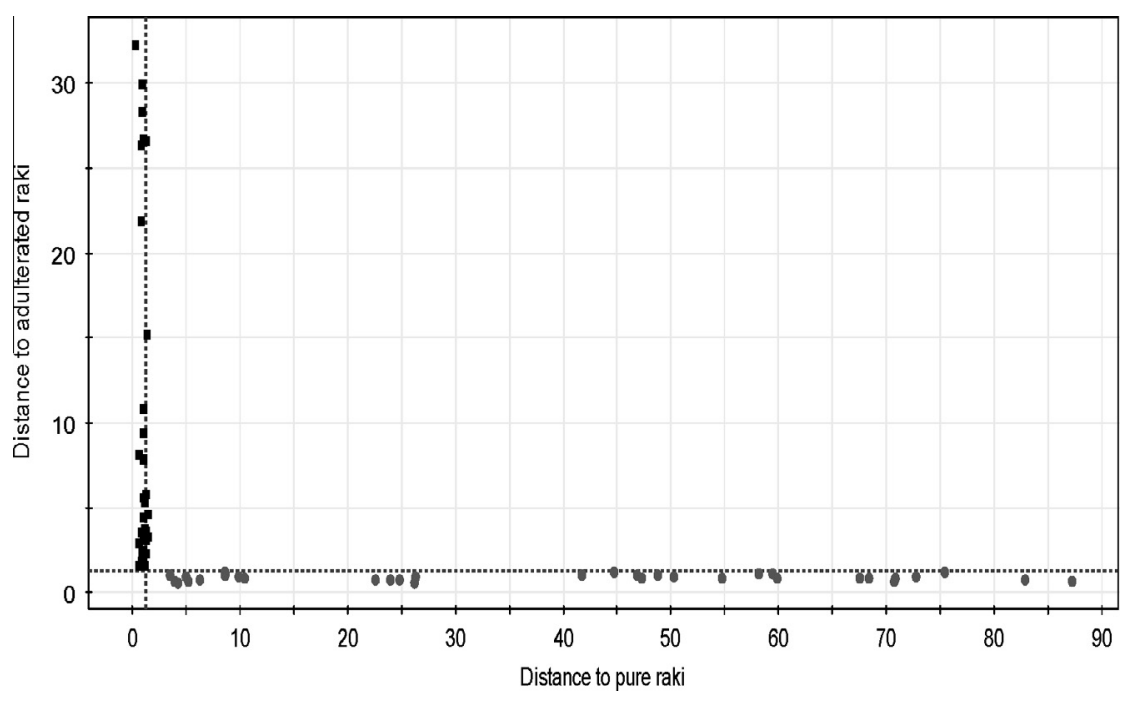

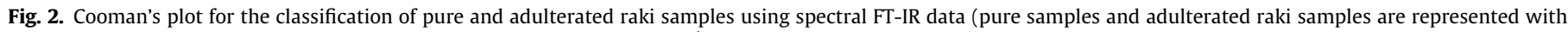
black and gray colours, respectively) (whole spectral range: $4000-650 \mathrm{~cm}^{-1}$ ). 
method is $0.5 \%$ ( $\mathrm{vol} / \mathrm{vol})$ while tolerance of people to methanol intoxication could be as low as $11.5 \mathrm{~g}$.

\section{References}

Anli, R. E., \& Bayram, M. (2010). Traditional aniseed-flavored spirit drinks. Food Reviews International, 26, 246-269.

Briandet, R., Kemsley, E. K., \& Wilson, R. H. (1996). Approaches to adulteration detection in instant coffees using infrared spectroscopy and chemometrics. Journal of the Science of Food and Agriculture, 71, 359-366.

Casela, M., Sinelli, N., Oliveri, P., Valentina Di Egidio, V. D. E., \& Silvia Lanteri, S (2010). Chemometrical strategies for feature selection and data compression applied to NIR and MIR spectra of extra virgin olive oils for cultivar identification. Talanta, 80, 1832-1837.

Di Maio, V. J., \& Di Maio, D. (2001). Forensic pathology. Boca Raton, FL: CRC Press.

Dixit, V., Tewari, J. C., Cho, B. K., \& Irudayaraj, J. M. K. (2005). Identification and quantification of industrial grade glycerol adulteration in red wine with Fourier transform infrared spectroscopy using chemometrics and artificial neural networks. Applied Spectroscopy, 12, 1553-1561.

Eke, M., Buyuk, Y., Dinc, A. H., \& Citici, I. (2007). Fatal alcohol intoxication amongst forensic autopsy cases in Ankara (2001-2004). Journal of Forensic Medicine, 21, 25-30.

Eriksson, L., Johanson, E., \& Wold, N. K. (2001). Multivariate data analysis. Principals and applications. Umea, Sweden: Umetrics AB.

Esbensen, K. H., Guyot, D., Westad, F., \& Houmøller, L. P. (2002). Multivariate data analysis: In practice: An introduction to multivariate data analysis and experimental design. Oslo, Norway: CAMO Software.

Fernandez, K., \& Agosin, E. (2007). Quantitative analysis of red wine tannins using Fourier-transform mid-infrared spectroscopy. Journal of Agricultural and Food Chemistry, 55, 7294-7300.

Fidan, I., Denli, Y., \& Anli, R. E. (1996). A research on the methanol content of raki produced in Turkey. Gida, 21, 415-418.

Gulmen, M. K., Meral, D., Hilal, A., Akcan, R., \& Cekin, N. (2006). Methanol intoxication in Adana, Turkey. Toxicology Mechanism and Methods, 16, 353-357.

Gurdeniz, G., \& Ozen, B. (2009). Detection of adulteration of extra-virgin olive oil by chemometric analysis of mid-infrared spectral data. Food Chemistry, 116, 519-525.

Kapur, B. M., Vandenbroucke, A. C., Adamchik, Y., Lehotay, D. C., \& Carlen, P. L. (2007). Formic acid, a novel metabolite of chronic ethanol abuse, causes neurotoxicity, which is prevented by folic acid. Alcoholism: Clinical and Experimental Research, 31, 2114-2120.

Kelly, J. F. D., \& Downey, G. (2005). Detection of sugar adulterants in apple juice using Fourier transform infrared spectroscopy and chemometrics. Journal of Agricultural and Food Chemistry, 53, 3281-3286.
Lachenmeier, D. W. (2007). Rapid quality control of spirit drinks and beer using multivariate data analysis of Fourier transform infrared spectra. Food Chemistry, $101,825-832$

Petrakis, P., Touris, I., Liouni, M., Zervou, M., Kyrikou, I., Kokkinofta, R., et al. (2005). Authenticity of the traditional Cypriot spirit 'zivania' on the basis of ${ }^{1} \mathrm{H}$ NMR spectroscopy diagnostic parameters and statistical analysis. Journal of Agricultural and Food Chemistry, 53, 5293-5303.

Polshin, E., Aernouts, B., Saeys, W., Delvaux, F., Delvaux, F. R., Saison, D., et al. (2011). Beer quality screening by FT-IR spectrometry: Impact of measurement strategies, data pre-processing and variable selection algorithms. Journal of Food Engineering, 106, 188-198.

Pontes, M. J. C. Santos, S. R. B., Araujo, M. C. U., Almeida, L. F. Lima, R. A. C., Gaiao, E. N., et al. (2006). Classification of distilled alcoholic beverages and verification of adulteration by near infrared spectrometry. Food Research International, 39, $182-189$.

Sadecka, J., Tothova, J., \& Majek, P. (2009). Classification of brandies and wine distillates using front face fluorescence spectroscopy. Food Chemistry, 117, 491-498.

Savchuk, S. A., Vlasov, V. N., Appolonova, S. A., Arbuzov, V. N., Vedenin, A. N., Mezinov, A. B., et al. (2001). Application of chromatography and spectrometry to the authentication of alcoholic beverages. Journal of Analytical Chemistry, 56, 214-231.

Sivakesa, S., \& Irudayaraj, J. (2001). Detection of inverted beet sugar adulteration of honey by FTIR spectroscopy. Journal of the Science of Food and Agriculture, 81, 683-690.

Tamaki, Y., \& Mazza, G. (2011). Rapid determination of lignin content of straw using Fourier transform mid-infrared spectroscopy. Journal of Agricultural and Food Chemistry, 59, 504-512.

TAPDK. (2010). <http://www.tapdk.gov.tr/TAPDK_Faaliyet.pdf> Last accessed 1.06.11.

Tarantilis, P. A., Troianou, V. E., Pappas, C. S., Kotseridis, Y. S., \& Polissiou, M. G. (2008). Differentiation of Greek red wines on the basis of grape variety using attenuated total reflectance Fourier transform infrared spectroscopy. Food Chemistry, 11, 192-196.

Turkish Food Codex. (2005). Communiqué on distilled alcoholic beverages. No: 2005/11. <http://www.kkgm.gov.tr/TGK/Teblig/2005-11.html> Last accessed 1.06.11.

Versari, A., Parpinello, G. P., Scazzina, F., \& Rio, D. D. (2010). Prediction of total antioxidant capacity of red wine by Fourier transform infrared spectroscopy. Food Control, 21, 786-789.

Yaycı, N., \& Inanici, M. A. (2005). Methyl alcohol intoxication. Türkiye KlinikleriJournal of Forensic Medicine, 2, 101-108.

Yilmaztekin, M., \& Cabaroglu, T. (2011). Confirmatory method for the determination of volatile congeners and methanol in Turkish raki according to European Union Regulation (EEC) No. 2000R2870: Single-laboratory validation. Journal of AOAC International, 94, 611-617. 